Proyecciones Journal of Mathematics

Vol. 30, $\mathrm{N}^{o}$ 1, pp. 59-64, May 2011.

Universidad Católica del Norte

Antofagasta - Chile

\title{
On graphs whose chromatic transversal number
} is two

\author{
S.K.Ayyaswamy \\ Sastra University, India \\ and \\ C. Natarajan \\ Sastra University, India
}

Received : October 2010. Accepted : February 2011

\begin{abstract}
In this paper we characterize the class of trees, block graphs, cactus graphs and cubic graphs for which the chromatic transversal domination number is equal to two.
\end{abstract}

Keywords : Domination number; chromatic transversal domination number; double star; cubic graphs; block graphs; cactus graphs.

AMS subject classification : 05C69. 


\section{Introduction}

Let $G=(V, E)$ be a simple graph of order $p$. A vertex $v$ of $G$ is a critical vertex if $\chi(G-v)<\chi(G)$, where $\chi(G)$ is the chromatic number of $G$. If every vertex of $G$ is a critical vertex, then $G$ is called a vertex critical graph. A subset $D \subset V$ is a dominating set, if every $v \in V-D$ is adjacent to some $u \in D$. The domination number $\gamma=\gamma(G)$ is the minimum cardinality of a dominating set of $G$. A dominating set $D$ is called a chromatic transversal dominating set (ctd-set) if $D$ has non empty intersection with every color class of every chromatic partition of $G$. The chromatic transversal domination number $\gamma_{c t}=\gamma_{c t}(G)$ is the minimum cardinality of a ctd-set of $G$. The parameter $\gamma_{c t}$ for a few well known graphs was computed by L.Benedict et al. [1]. For example, if $G$ is a vertex critical graph of order $p$, then $\gamma_{c t}(G)=p$.

By a double star we mean a tree obtained by joining the centers of two stars $K_{1, m}$ and $K_{1, n}$ by an edge. If we subdivide the edge connecting the centers of two stars, then it is called a double star with one subdivision. Similarly, a double star with two subdivisions is defined. The diameter of a graph $G$ is the length of the longest path in $G$ and is denoted by $\operatorname{diam}(G)$. A vertex $v$ of a connected graph $G$ is said to be a cutvertex if $G-v$ is no longer connected. A connected subgraph $B$ of $G$ is a block, if $B$ has no cutvertex and every subgraph $B^{\prime} \subset G$ with $B \subset B^{\prime}$ and $B \neq B^{\prime}$ has at least one cutvertex. A block $B$ of $G$ is called an end block, if $B$ contains at most one cutvertex of $G$; such a cutvertex is called an end block cutvertex. A graph $G$ is called a block graph, if every block $G$ is a complete graph. A graph $G$ is called a cactus graph if every edge of $\mathrm{G}$ is in at most one cycle of G. A graph $G$ is said to be a cubic graph if it is 3-regular. $\Delta(G)=\max \{\operatorname{deg}(v): v \in V(G)\}$. A support vertex in $G$ is one which is adjacent to a leaf.

Theorem 1.0.1. [2] Let $G$ be a connected bipartite graph of order $p \geq 3$ with partition $\left(V_{1}, V_{2}\right)$ of $V(G)$, where $\left|V_{1}\right| \leq\left|V_{2}\right|$. Then $\gamma_{c t}(G)=\gamma(G)+1$ if and only if every vertex in $V_{1}$ has at least two pendant neighbors.

Theorem 1.0.2. [2] For a tree $T, \gamma_{c t}(T)=\gamma(T)+1$ if and only if either $T$ is $K_{2}$ or $T$ satisfies the condition that whenever $v$ is a support vertex, then each vertex $w$ with $d(v, w)$ even is also a support vertex and each support vertex has at least two pendant neighbors. Otherwise $\gamma_{c t}(T)=\gamma(T)$. 


\section{Characterization}

\subsection{Trees}

Lemma: 2.1.1. For a tree $T, \gamma(T)=2$ if and only if $T$ is one of the following:

(i) a double star

(ii) a double star with one subdivision

(iii) a double star with two subdivisions.

Proof: Assume that $\gamma(T)=2$.

Claim: $\operatorname{diam}(T) \leq 5$.

If not, let $P$ be the largest path in $T$ with length greater than 5 . Then $\gamma(P) \geq 3$ where $\gamma(P)$ refers to the domination number of the path $P$. Without loss of generality assume that $\gamma(P)=3$ and let $D=\left\{x_{1}, x_{2}, x_{3}\right\}$ be a $\gamma$-set of $P$.

Now, take any $\gamma$-set $S=\{x, y\}$ of $T$. If $x$ or $y$ is not in $P$, then a cycle will be formed with one of the vertices of $D$. In fact, if $x=x_{1}$ and $y$ is not in $P$, then $x_{3}$ must be adjacent to $y$ and at least one of the neighbors of $x_{3}$, say $u$, will be adjacent to $y$ so that the vertices $x_{3}, y, u$ form a cycle. Thus $x, y \in P$. But if $x, y \in P$ then at least one of the vertices of $D$ will not be dominated by $S$, contradicting the assumption that $\gamma(T)=2$.

Case 1. Let $\operatorname{diam}(T)=3$ and $P_{4}: u_{1} u_{2} u_{3} u_{4}$ be the longest path in $T$. If $S=\{x, y\}$ is a $\gamma$-set of $T$, then as argued earlier, $S \subset V\left(P_{4}\right)$. As $P_{4}$ is the longest path in $T$ it follows that $u_{1}$ and $u_{4}$ are pendant vertices in $T$. If $x=u_{1}$ and $y=u_{4}$, then $T$ is a path $P_{4}$. If $x=u_{1}$ and $y=u_{3}$, then $T$ is a double star with $K_{1, t}$ at $u_{3}$. Similarly, we get a double star if $x=u_{2}$ and $y=u_{4}\left(\right.$ or if $x=u_{2}$ and $\left.y=u_{3}\right)$.

Case 2. Let $\operatorname{diam}(T)=4$ and $P_{5}: u_{1} u_{2} u_{3} u_{4} u_{5}$ be the longest path in $T$ with $u_{1}$ and $u_{5}$ as pendant vertices in $T$. Then for any $\gamma$-set $S=\{x, y\}$ of $T$, we have $S \subset V\left(P_{5}\right)$. We claim that $x=u_{2}$ and $y=u_{4}$. Suppose $x=u_{3}$ and $y=u_{4}$. Then $u_{1}$ will not be dominated by $S$. Similarly, the other possibilities for $x$ and $y$ except $x=u_{2}$ and $y=u_{4}$. Thus $T$ is a double star 
with one subdivision.

Case 3. Let $\operatorname{diam}(T)=5$ and $P_{6}: u_{1} u_{2} u_{3} u_{4} u_{5} u_{6}$ be the longest path in $T$. Then any $\gamma$-set $S=\{x, y\}$ of $T$ is a subset of $V\left(P_{6}\right)$ and as argued earlier $x=u_{2}$ and $y=u_{4}$ making $T$ a double star with two subdivisions.

The converse is obvious.

Theorem 2.1.1. For a tree $T, \gamma_{c t}(T)=2$ if and only if $T$ is one of the following:

(i) a double star

(ii) a double star with two subdivisions

(iii) a star graph.

Proof: Assume that $\gamma_{c t}(T)=2$. According to Theorem 1.0.2, $\gamma_{c t}(T)$ is either $\gamma(T)$ or $\gamma(T)+1$. If $\gamma_{c t}(T)=\gamma(T)$, then $\gamma(T)=2$ and so by Lemma 2.1.1, $T$ is a double star or a double star with one subdivision or a double star with two subdivisions. But $T$ cannot be a double star with one subdivision in view of Theorem 1.0.2.

If $\gamma_{c t}(T)=\gamma(T)+1$, then $\gamma(T)=1$ and so $T$ is a star graph.

The converse is obvious.

\subsection{Block graphs}

Proposition: 2.2.1. For a block graph $G, \gamma_{c t}(G)=2$ if and only if $G$ is a star graph.

Proof: Assume that $\gamma_{c t}(G)=2$. Let $K_{n}$ be a maximal clique with maximum number of cutvertices. Then one can show that $\gamma_{c t}(G)=n+\gamma\left(G^{\prime}\right)$ where $G^{\prime}=G-V\left(K_{n}\right)-L$ and $L$ is the set of all leaves with supports at some vertices of $K_{n}$. Then $\gamma_{c t}(G)=2$ if and only if $n=2$ and $\gamma\left(G^{\prime}\right)=0$ or $n=1$ and $\gamma\left(G^{\prime}\right)=1$. In either case $G$ is a star graph.

The converse is obvious.

Note: For a block graph $G$, one can easily verify that $\gamma(G)=2$ if and only if $G$ has exactly two end vertices and at most two internal cut vertices. 


\subsection{Cubic graphs}

Proposition: 2.3.1. For a connected cubic graph $G$ of order $p, \gamma_{c t}(G)=2$ if and only if $p \leq 8$.

Proof: Let $G$ be a cubic graph with $\gamma_{c t}(G)=2$. Then since $\gamma_{c t}(G) \geq \chi(G)$, $\chi(G)=2$. That is $G$ is a bipartite graph. Therefore by Theorem 1.0.1, $\gamma_{c t}(G)=\gamma(G)$ and so $\gamma(G)=2$. But then we have $\frac{p}{1+\Delta(G)} \leq 2$ which implies $\frac{p}{4} \leq 2$. That is $p \leq 8$. Conversely, if $G$ is a cubic graph with $p \leq 8$, one can easily verify that $\gamma_{c t}(G)=2$.

This proves the result.

\subsection{Cactus graphs}

Proposition: 2.4.1. If $G$ is a cactus graph with at least one cycle, then $\gamma_{c t}(G)=2$ if and only if $G$ is either $C_{4}$ with at most two support vertices that are adjacent or $C_{6}$ with a pair of support vertices $u_{i}$ and $u_{j}$ where $j=i+3(\bmod 6)$ if $V\left(C_{6}\right)=\left\{u_{0}, u_{1}, \ldots, u_{5}\right\}$.

Proof: Let us assume that $\gamma_{c t}(G)=2$. If $G$ has an odd cycle, $\chi(G)=3$ and so $\gamma_{c t}(G) \geq 3$, a contradiction. Therefore $G$ cannot have an odd cycle.

Suppose $G$ has an even cycle of length greater than or equal to 8 . Then $\gamma_{c t}(G)=\left\lceil\frac{8}{3}\right\rceil=3$, which is a contradiction. Therefore $G$ has an even cycle of length 4 or 6 . Furthermore $G$ is unicyclic. If not, $\gamma_{c t}(G) \geq \gamma(G) \geq 3$.

Case 1. Let $G$ be a unicyclic graph with $C_{4}$, a cycle of length 4 . Let $X$ be the set of all vertices of degree 2 in $C_{4}$. Now $\gamma_{c t}(G)=2$ implies $|X| \geq 2$. If $|X|=4, G$ is just $C_{4}$. If $|X|=3, G$ is $C_{4}$ with one support vertex. Similarly, if $|X|=2, G$ is $C_{4}$ with two support vertices and as $\gamma_{c t}(G)=2$, these support vertices are adjacent.

Case 2. Let $G$ be a unicyclic graph with $C_{6}$, cycle of length 6 .

The proof of this case is just similar to Case 1 except that two support vertices require to be of distance 3 to form a $\gamma_{c t}$-set of $G$.

The converse is obvious. 
Acknowledgement: The authors wish to thank the anonymous referees for their valuable suggestions.

\section{References}

[1] L. Benedict Michaelraj, S. K. Ayyaswamy and S. Arumugam, Chromatic transversal domination in graphs; J. Comb. Math. Comb. Comput. (Accepted for publication)

[2] L. Benedict Michaelraj, A Study on Chromatic Transversal Domination in graphs; (Ph.d Thesis), Bharathidasan University, Trichy, Tamilnadu, India, April 2008.

[3] T. W. Haynes, S. T. Hedetniemi and P. J. Slater, Fundamentals of Domination in graphs, Marcel Dekker, New York, (1998).

\section{S. K. Ayyaswamy}

Department of Mathematics, SASTRA University,

Thanjavur - 613 401,

Tamilnadu,

India

e-mail : sjcayya@yahoo.co.in

and

\section{Natarajan}

Department of Mathematics,

SASTRA University,

Thanjavur - 613 401,

Tamilnadu,

India

e-mail : natarajan_c@maths.sastra.edu 